\title{
Paediatric crossword puzzle 24
}

\author{
Manouri P. Senanayake ${ }^{1}$
}

Sri Lanka Journal of Child Health, 2014; 43(3): 183

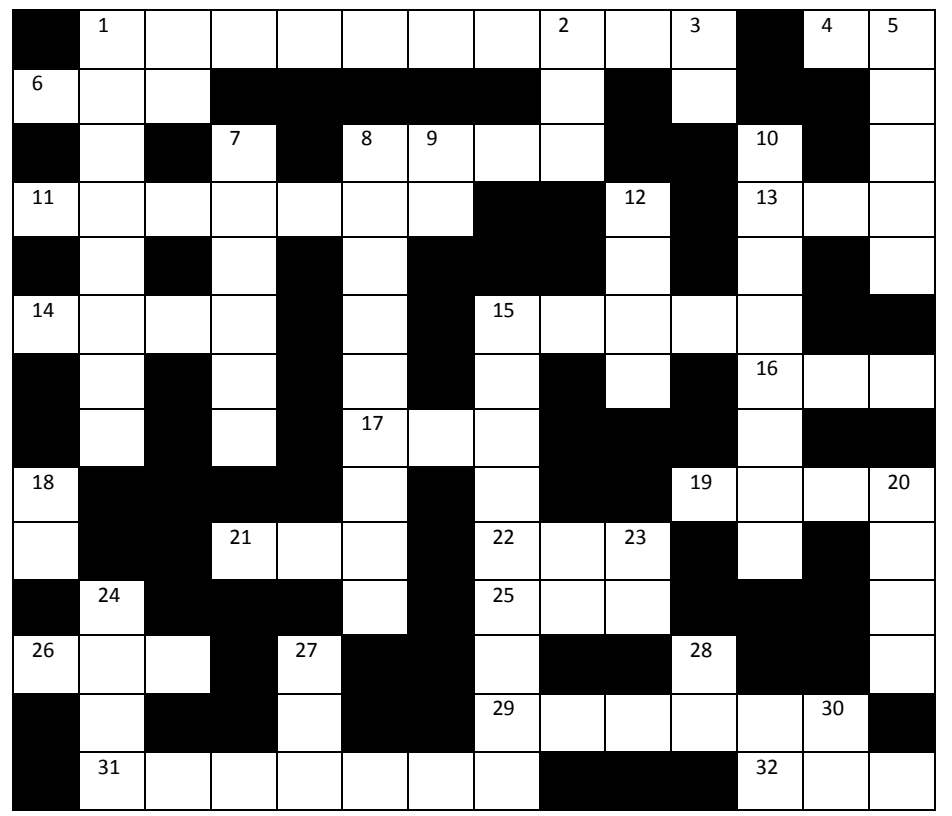

$\underline{\text { Across }}$

1. Largest known protein coding gene

4. Poisonous gas in exhaust fumes

6. Source of Dirofilariasis

8. Furosemide is a --------- diuretic

11. Form of cell division

13. An important tool for screening of fetus (abbrv)

14. Rate of transmission of congenital toxoplasmosis is highest in gestation

15. Hairy-------- is transmitted on Y chromosome

16 . Benign self limiting respiratory condition in newborn (abbrv)

17. Strength of folic acid tablet currently available is ------------mg

19. Late onset cataract causing------ syndrome

21. Best parameter for screening childhood obesity (abbrv)

22. Vaccine to be withdrawn as a part of "Polio End Game"

25. Number of X chromosomes in Klinefelter syndrome

26. Mother to baby transmission of virus is mostly intra partum (abbrv)

29. Body not secured by blood brain barrier 31. Name of pediatrician who first advocated neonatal screening

32. Oncogenic virus preventable by vaccination

$\underline{\text { Down }}$

1. Autosomal ------- transmission causes three fourth of affected offspring when parents are heterogeneous 2. Sequencing of the human genome (abbrv)

3. A medically useful free radical

5. Above $95^{\text {th }}$ centile, weight for height

7. Sign of "climbing up on one's own thighs"

8. Gaucher disease is an inherited

9. Latin for "an opening"

10. Alteration of gene during division

12. Transient tachypnoea of newborn is due to delayed clearance of -------------- fluid

15. Intersex termed a hermaphrodite is specified according to

18. Synonymous with somatotrophin (abbrv)

20. Landmark age for precocious puberty in boys

23. Visiting obstetrician

24. A chromosome that is rejoined following deletion of two short arms

27. Maternal condition causing IUGR

28 "id est." or "that is" (abbrv)

30. Procedure for obtaining CSF (abbrv) 